\title{
Defibrination syndrome with defective thrombin- fibrinogen reaction reversible by protamine
}

\author{
A. L. BLOOM AND NORAH CAMPBELL \\ From the Institute of Pathology, Cardiff Royal Infirmary, and the Department of Obstetrics and Gynaecology, \\ Maternity Hospital and Royal Infirmary, Cardiff
}

SYNOPSIS A patient with the obstetric defibrination syndrome successfully treated with human fibrinogen is described. The blood not only contained low levels of fibrinogen capable of clotting but also contained an inhibitor of the thrombin-fibrinogen reaction. This was neutralized in vitro with protamine but it was not heparin. Neither abnormality was detected by a latex agglutination test for plasma fibrinogen. The use of this test does not therefore exclude the diagnosis of the $\stackrel{\circ}{\triangle}$ defibrination syndrome.

Abnormal coagulation of the blood due to acute defibrination occurring in patients with concealed accidental haemorrhage and certain other complications of pregnancy is an uncommon but dangerous emergency. The defibrination syndrome is not confined to obstetric cases and may occur, for example, during thoracic surgery, especially with extracorporeal circulation, during haemolytic transfusion reactions, and in patients with carcinoma of the prostate or pancreas. The syndrome is so named because the outstanding abnormality is the absence or low level of plasma fibrinogen due to intravascular activation of the coagulation or fibrinolytic systems, but greater or lesser degrees of thrombocytopenia and deficiency of factor V and factor VIII may also occur as a result of the same mechanisms (Sharp, Howie, Biggs, and Methuen, 1958). Surviving fibrinogen sometimes reacts abnormally to thrombin and may not contribute effectively to haemostasis. In one case recorded by Baker and Jacob (1960) this defect was corrected in vitro by protamine.

A latex agglutination test available commercially is widely used as a rapid bedside technique to detect critical levels of plasma fibrinogen. This test is based on the assumption that the agglutination by blood of latex particles coated with antihuman fibrinogen indicates a haemostatic level of fibrinogen. The object of this paper is to report a patient with the obstetric defibrination syndrome whose plasma exhibited a defective thrombin-fibrinogen reaction reversible by protamine as well as a low level of clottable fibrinogen. Neither abnormality was

${ }^{1}$ Present address: Department of Obstetrics and Gynaecology, Hammersmith Hospital, Du Cane Road, London, W.12.

Received for publication 12 May 1965. detected by using a latex antihuman fibrinogen $\frac{\Phi}{3}$ reagent.

\section{CASE REPORT}

The patient was a 29-year-old married woman. Her first four pregnancies had been uneventful but the fifth had terminated spontaneously at the fifteenth week. There was no history of bleeding tendency or other relevant illness. She was admitted to the Maternity Hospital, $\frac{\mathbb{D}}{8}$

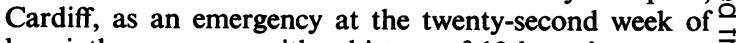
her sixth pregnancy with a history of 10 hours' constant $\overrightarrow{\vec{O}}$ lower abdominal pain. She had ceased to feel foetal movements during this time and had noticed slight vaginal blood loss for one hour.

Physical examination revealed that she was pale but not shocked. Blood pressure was $120 / 85 \mathrm{~mm} . \mathrm{Hg}$, pulse rate $86 / \mathrm{min}$., and temperature $36^{\circ} \mathrm{C}$. The uterus, tense and slightly tender, corresponded in size to a 34-week pregnancy. No foetal parts were palpable and the foetal 8 heart could not be heard. A diagnosis of probable accidental antepartum haemorrhage was made.

The haemoglobin level was $7.9 \mathrm{~g}$. per $100 \mathrm{ml}$. and it was noted that blood taken for grouping, cross matching, $\frac{7}{0}$ and coagulation studies did not clot. Bruising occurred at the site of venepuncture. Shortly after admission her N condition deteriorated with falling blood pressure and blood transfusion was commenced. Sudden loss of blood $N$ from the vagina occurred and this blood did not clot. $A \underset{\omega}{N}$ clinical diagnosis of the defibrination syndrome was made 0 and a solution of human fibrinogen prepared. Meanwhile laboratory studies (see below) had confirmed the clinical diagnosis and $2.3 \mathrm{~g}$. of fibrinogen was administered $\stackrel{\infty}{\infty}$ intravenously. Her platelet count was noted to be reduced ? to 90,000 per c.mm. but the thromboplastin generation $\frac{0}{0}$ screening test (Hicks and Pitney, 1957) revealed no $\frac{\mathrm{C}}{\mathbb{D}}$ significant deficiency of factor VIII or factor V.

Following this treatment her condition improved $\mathbb{D}$ sufficiently to allow vaginal examination. The cervix was 
found to be taken up and two fingers dilated. The forewaters were ruptured and several ounces of bloodstained liquor allowed to drain. Treatment was continued with a further $2 \cdot 2 \mathrm{~g}$. of fibrinogen and blood transfusion. By this time, some three to four hours after admission, the patient was established in labour and her clinical condition was satisfactory. Examination of her blood revealed an increased fibrinogen titre and although the presence of an anticoagulant neutralized in vitro by protamine had already been demonstrated her condition did not warrant administration of this drug.

Spontaneous delivery of a small female foetus occurred nine hours after admission. Loss with the placenta totalled $63 \mathrm{oz}$. of altered blood containing no clots; the appearances were consistent with concealed accidental haemorrhage. Further loss of blood was trivial and apart from a slight fall of blood pressure immediately after delivery the patient made a good recovery. Laboratory tests 10 hours after delivery showed a normal fibrinogen level. In all $4.5 \mathrm{~g}$. of fibrinogen and 6 pints of blood were administered during labour and a further 3 pints of blood were given later to correct anaemia. She was discharged from hospital on the tenth day after delivery when her haemoglobin level was $11.8 \mathrm{~g}$. per $100 \mathrm{ml}$.

\section{LABORATORY STUDIES}

METHODS Nine parts of blood were taken into 1 part of $3.8 \% \mathrm{w} / \mathrm{v}$ sodium citrate solution and plasma obtained by centrifuging at 3,000 r.p.m. for 10 minutes. Plasma fibrinogen levels were assessed by the fibrinogen titre technique (Sharp et al., 1958). In some experiments protamine sulphate solution, $0.1 \mathrm{ml}$. of a $100 \mathrm{mg} . \%$ solution, or saline as control, was added to each tube. A fibrinogen titre of $1: 32$ or over indicates a plasma fibrinogen level of over $100 \mathrm{mg} . \%$.

The plasma fibrinogen level was also assessed by means of a latex antihuman fibrinogen reagent (F I test, Hyland Laboratories) used on whole blood according to the manufacturer's instructions. Agglutination of the latex is alleged to indicate a plasma fibrinogen level of over $100 \mathrm{mg} . \%$.

The thrombin clotting time was determined by mixing $0.2 \mathrm{ml}$. of plasma with $0.2 \mathrm{ml}$. saline and noting the clotting time at $37^{\circ} \mathrm{C}$. on the addition of $0.1 \mathrm{ml}$. of a solution of bovine thrombin (Parke Davis). The strength of thrombin was adjusted to give a clotting time of about
15 seconds with normal plasma. In some experiments protamine sulphate was substituted for the saline, and in others mixtures of normal and patient's plasma were tested. Details of these and other experimental methods are described below.

Three samples of the patient's blood were tested: before and after fibrinogen therapy and on the next day some 10 hours after delivery. Most of the tests were performed immediately, but some were done on plasma stored for a short time at $-20^{\circ} \mathrm{C}$. Repetition of original tests on such stored plasma showed no appreciable change.

\section{RESULTS}

PLASMA FIBRINOGEN LEVElS The initial sample was incoagulable with thrombin and the fibrinogen titre was thus nil, no clot forming in any dilution of the plasma tested, i.e., up to $1: 128$. The latex test, however, performed on the ward and in the laboratory with different batches of reagents indicated a haemostatic level of fibrinogen of over $100 \mathrm{mg} . \%$. The introduction of protamine into the fibrinogen titre test resulted in the formation of clot to a titre of 1 : 16 (Table I). This could not be increased by increasing the concentration of protamine. After fibrinogen therapy clot was formed to a titre of $1: 16$, and with protamine added to a normal level of $1: 128$. On the next day, after delivery, the fibrinogen titre was normal even without added protamine.

\section{TABLE I}

FIBRINOGEN TITRE OF THE PATIENT'S PLASMA WITH AND WITHOUT THE ADDITION OF PROTAMINE

\begin{tabular}{lcc} 
Sample Tested & \multicolumn{2}{l}{ Fibrinogen Titre } \\
\cline { 2 - 3 } & Without Protamine & With Protamine \\
\hline Initial sample & Nil & $1: 16$ \\
After fibrinogen therapy & $1: 16$ & $1: 128$ \\
After delivery & $1: 128$ & $1: 128$
\end{tabular}

THROMBIN-FIBRINOGEN REACTION The initial sample not only showed an infinitely prolonged thrombin clotting time, but it prolonged the thrombin clotting time of an equal volume of normal plasma (Table II).

\section{TABLE II}

THROMBIN CLOTTING TIMES OF PATIENT'S, NORMAL, AND EQUAL VOLUMES OF PATIENT'S AND NORMAL PLASMA WITH AND WITHOUT PROTAMINE

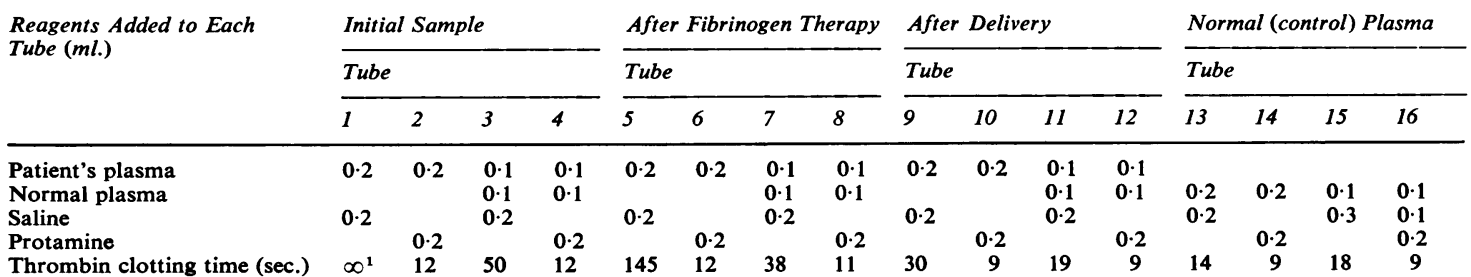

${ }^{1}$ After incubation for $180 \mathrm{sec}$. addition of protamine caused instantaneous clotting. 
Protamine corrected these abnormalities and moreover when added to plasma a short time after the addition of thrombin it caused instantaneous clot formation. Toluidine blue was also effective in correcting the thrombin clotting time. The changes were less marked in the sample taken after fibrinogen therapy and had almost disappeared by the next day.

These findings suggested the presence of an inhibitor of the thrombin-fibrinogen reaction. This was confirmed by measuring the thrombin clotting time of mixtures of patient's and normal plasma in various proportions. Because of shortage of plasma this experiment was performed only on the sample taken after fibrinogen therapy. The results are shown in Table III. The thrombin clotting time was

TABLE III

THROMBIN CLOTTING TIME OF PATIENT'S AND NORMAL PLASMA IN VARYING PROPORTIONS

$\begin{array}{lrrrrrrr}\text { Parts patient's plasma } & 10 & 9 & 8 & 5 & 2 & 1 & 0 \\ \text { Parts normal plasma } & 0 & 1 & 2 & 5 & 8 & 9 & 10 \\ \text { Thrombin clotting time (secs.) } & 130 & 125 & 67 & 38 & 18 & 17 & 16\end{array}$

appreciably prolonged only in mixtures containing an excess of patient's plasma. A small proportion had relatively little effect. An attempt was made to adsorb the inhibitor with barium sulphate. Table IV shows the result of thrombin clotting time estimations of mixtures of normal oxalated plasma with equal volumes of adsorbed and non-adsorbed normal, heparinized (0.25 units per $\mathrm{ml}$.), and patient's oxalated plasma. Adsorption was carried out for 15 minutes at $37^{\circ} \mathrm{C}$., with $50 \mathrm{mg}$. of washed radiographic barium sulphate to $0.5 \mathrm{ml}$. plasma. Barium sulphate removed heparin completely from plasma but had no effect on the inhibitor under study.

\section{TABLE IV}

EFFECT OF BARIUM SULPHATE ADSORPTION ON THE INHIBITORY EFFECT OF THE PATIENT'S AND HEPARINIZED PLASMA ON THE THROMBIN CLOTTING TIME OF NORMAL

\begin{tabular}{|c|c|c|c|c|c|c|}
\hline \multirow{3}{*}{ Plasma Tested } & \multirow{2}{*}{\multicolumn{6}{|c|}{$\begin{array}{l}\text { PLASMA } \\
\text { Tube }\end{array}$}} \\
\hline & & & & & & \\
\hline & 1 & 2 & 3 & 4 & 5 & 6 \\
\hline $\begin{array}{l}\text { Normal plasma (ml.) } \\
\text { Normal adsorbed plasma } \\
(\mathrm{ml} .)\end{array}$ & $0 \cdot 2$ & $\begin{array}{l}0 \cdot 1 \\
0 \cdot 1\end{array}$ & $0 \cdot 1$ & 0.1 & $0 \cdot 1$ & $0 \cdot 1$ \\
\hline $\begin{array}{l}\text { Patient's plasma }(\mathrm{ml} .)^{1} \\
\text { Patient's adsorbed plasma } \\
(\mathrm{ml} .)^{1}\end{array}$ & & & 0.1 & $0 \cdot 1$ & & \\
\hline $\begin{array}{l}\text { Heparinized plasma (ml.) } \\
\text { Adsorbed heparinized } \\
\text { plasma (ml.) } \\
\text { Thrombin clotting time }\end{array}$ & & & & & 0.1 & $0 \cdot 1$ \\
\hline $\begin{array}{l}\text { (sec.) } \\
\text { Sample taken after fibrino }\end{array}$ & $\begin{array}{l}15 \\
\text { gen th }\end{array}$ & 15 & 38 & 38 & $180+$ & 16 \\
\hline
\end{tabular}

FIBRINOLYSIS Plasma was examined for excessive fibrinolysis simply by observing the clots formed in fibrinogen titre tests incubated at $37^{\circ} \mathrm{C}$. over 24 hours. Assays of plasminogen were not performed 0 ? but mixtures of equal volumes of patient's and normal plasma (to supply plasminogen and clottable fibrinogen) were set up and observed. In no sample did lysis of clots occur within 24 hours.

\section{DISCUSSION}

The main features of the present case were the presence of low levels of fibrinogen in the plasma as measured by the fibrinogen titre test and inhibition $\overrightarrow{0}$ of the thrombin-fibrinogen reaction reversible by protamine and toluidine blue. These abnormalities $\infty$ developed in a patient suffering from concealed accidental haemorrhage and appear to be identical ő to those described in another case by Baker and $\mathrm{O}$ Jacob (1960). Their patient developed a severe and ultimately fatal haemorrhagic tendency during $z$ labour. Her blood would not coagulate and her plasma, except in low proportion, prolonged the calcium clotting time of normal plasma. The defect was corrected by protamine and all stages of coagulation before the thrombin-fibrinogen reaction were normal.

The nature of the inhibitory action of the patient's plasma in the thrombin-fibrinogen reaction was not established with certainty in the present study. A heparin-like anticoagulant was unlikely as the inhibitor was not adsorbed by barium sulphate. The fact that protamine caused instantaneous clotting of a plasma-thrombin mixture suggests that thrombin had already acted on fibrinogen and supports the suggestion of Baker and Jacob (1960) that the defect is due to the presence of abnormal or altered fibrinogen which not only fails to polymerize 웅 normally but when present in sufficient quantity interferes with the polymerization of the normal fibrin monomer. The corrective effect of protamine $\delta$ and toluidine blue may be related to the accelerating effect which these substances exert on the normal $\subseteq$ thrombin-fibrinogen reaction.

Although fibrinogen loss is often a cardinal feature of the obstetric defibrination syndrome, N Sharp and his co-workers (1958) have shown that defective thrombin-fibrinogen reaction is also $\mathbb{O}$ important. In three of four cases which they studied $\underset{\omega}{\mathrm{W}}$ plasma fibrinogen levels measured biochemically were within the haemostatic range yet were reduced 0

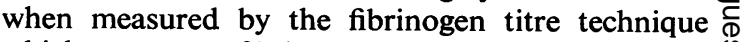
which measures fibrinogen clottable by thrombin. $\stackrel{\mathcal{Q}}{?}$ The thrombin clotting times were also prolonged 0 although not capable of correction by toluidine blue. This abnormality is not confined to obstetric cases. Prolongation of the thrombin clotting time, not due to heparin, has also been reported in the defibrin- 
ation syndrome following extracorporeal circulation (Von Kaulla and Swan, 1958; Gans and Krivit, 1962), with carcinoma of the pancreas (Rapaport and Chapman, 1959), and in experimental defibrination produced by the injection of tissue thromboplastin (Ratnoff and Conley, 1951; Hartmann, Conley, and Krevans, 1951). Perhaps the reactivity of fibrinogen to thrombin is altered during intravenous activation of the coagulation mechanism.

Excessive fibrinolysis is sometimes a feature of the defibrination syndrome and is thought by some workers (Soulier, Petit, and Le Bolloch, 1952) to be the primary factor in its pathogenesis. Sherry and his co-workers (Fletcher, Alkjaersig, and Sherry, 1962) detected prolonged thrombin clotting times after intravenous injection of streptokinase. They considered that the presence of fibrinogen breakdown products leads to defective fibrin polymerization but this was not corrected by protamine. A similar inhibitory phenomenon previously reported by Niewiarowski and Kowalski (1958), however, was reversed by protamine and toluidine blue and was not removed by barium sulphate. Although these findings resemble those of our patient, increased fibrinolysis was not demonstrated. It is possible, however, that fibrinolytic activity had disappeared by the time the patient was examined.

An alternative explanation for the abnormal thrombin-fibrinogen reaction is that the inhibitor, possibly altered fibrinogen, gained access to the inaternal circulation from the placental site. Consistent with this theory is the observation of Baker and Jacob (1960) that the inhibitor was also present in the infant's blood. We have no data on the thrombin-fibrinogen reaction of the cord blood in the present case but an identical abnormality can sometimes be detected in the blood of newborn infants after apparently normal delivery (Dr. J. T. Roberts, personal communication).

Baker and Jacob (1960) hesitated to classify their case within the general syndrome of obstetric defibrination because the plasma fibrinogen level was normal. This was measured biochemically as protein capable of clotting in plasma containing protamine. Sharp and his colleagues (1958) have shown that this assay may correlate poorly with the fibrinogen titre technique and they base their diagnosis of obstetric defibrination on the latter method. In our case the fibrinogen titre was reduced even in the presence of optimum concentrations of protamine. We consider that such a defect, developing, together with thrombocytopenia, in a patient with concealed accidental haemorrhage and responding to fibrinogen therapy, is compatible with the diagnosis of the obstetric defibrination syndrome. The response of the patient's plasma to protamine was unusual but may be detected more frequently if specially sought for. Thus a patient described by Baumann (1952) developed 'afibrinogenemia' after lobectomy. The blood clotted normally 15 minutes after the injection of protamine.

The diagnosis of defibrination is usually a matter of some urgency and depends upon the availability of rapid simple tests for the assessment of plasma fibrinogen levels and the thrombin-fibrinogen reaction. The latex agglutination test is widely used to detect the presence or absence of haemostatic level of fibrinogen. This test has certain possible disadvantages. Ferreira and Murat (1963) have shown that the products of fibrinolysis and fibrinogenolysis react immunologically with antihuman fibrinogen. There is thus the theoretical possibility that a positive latex test is merely demonstrating fibrin or fibrinogen breakdown products. Furthermore the test is not designed to detect an abnormal thrombinfibrinogen reaction and may react with abnormal non-clottable fibrinogen. The test was quite normal in our patient. We agree with Sharp and his colleagues that the fibrinogen titre test and the thrombin clotting time or one of its modifications are the best methods for detecting defibrination.

The adequate treatment of the defibrination syndrome depends to a large extent upon the availability of human fibrinogen. Our patient was treated with fibrinogen and recovered. Baker and Jacob (1960) were unable to obtain fibrinogen and their patient died. They did however try the effect of the intravenous injection of $150 \mathrm{mg}$. of protamine. This caused a slight improvement in coagulation and it was unfortunate that further supplies were not available. Protamine was not given to our patient because of the favourable response to fibrinogen and because of the possibility of triggering further defibrination by correcting the thrombin-fibrinogen reaction completely.

We would like to thank Mr. M. D. Arwyn Evans for permission to publish an account of this patient.

\section{REFERENCES}

Baker, S. J., and Jacob, E. (1960). J. clin. Path., 13, 214.

Baumann, J. (1952). Rev. Hémat., 7, 20.

Ferreira, H. C., and Murat, L. G. (1963). Brit. J. Haemat., 9, 299.

Fletcher, A. P., Alkjaersig, N., and Sherry, S. (1962). J. clin. Invest., 41, 896.

Gans, H., and Krivit, W. (1962). J. Amer. med. Ass., 179, 145.

Hartmann, R. C., Conley, C. L., and Krevans, J. R. (1951). J. clin. Invest., 30, 948.

Hicks, N. D., and Pitney, W. R. (1957). Brit. J. Haemat., 3, 227. Niewiarowski, S., and Kowalski, E. (1958). Rev. Hémat., 13, 320.

Rapaport, S. I., and Chapman, C. G. (1959). Amer. J. Med., 27, 144. Ratnoff, O. D., and Conley, C. L. (1951). Bull. Johns Hopk. Hosp., 88, 414.

Sharp, A. A., Howie, B., Biggs, R., and Methuen, D. T. (1958). Lancet, 2, 1309.

Soulier, J. P., Petit, P., and Le Bolloch, A. G. (1952). Rev. Hémat., 7, 48.

Von Kaulla, K. N., and Swan, H. (1958). J. thorac. Surg., 36, 519. 